\title{
Optimization analysis of XML embedded system based on J2ME platform
}

\author{
Ying Wang, Zequan Shi \\ Department of Information Engineering,Chongqing Institute of Engineering, Chongqing, 402260, \\ China
}

yingwang_2013@yeah.net

Keywords: embedded system; transplantation; modular

\begin{abstract}
In the optimization research of XML embedded system, the traditional method has disadvantages like poor stability and time-consuming. In order to make up for these shortcomings, the optimization method of XML embedded system based on J2ME platform is put forward, according to the characteristics of J2ME, such as free structure, modularity and scalability, through building cross compiler environment and relevant basic configuration, operation platform of J2ME is translated and established in XML embedded system, so as to reduce the workload of system itself and improve the stability of the system. Simulation results show that, the optimization method of XML embedded system based on J2ME platform have strong stability, and high work efficiency.
\end{abstract}

\section{Introduction}

Nowadays, the electronic industry is developing rapidly, J2ME technology is utilized to enhance performance of consumer electronic products, the design of J2ME system structure must be diverse, specific standard is achieved based on the application deployment in actual situation (1). The focus in J2ME technology is to build up the J2ME platform, but the technology is stronger, and more cumbersome, must comply with the basic standards which is closely linked to the underlying hardware and software, and only if reflect the function of Java virtual machine of J2ME specification at a particular platform, the errors between the underlying hardware and software can be eliminated for J2ME applications, so as to achieve the object to create a good environment for the operation (2-4). In the research of XML embedded system optimization, with the mminiXML to optimize XML embedded system in routine is tedious, and have huge workload, poor reliability. In view of the above question, the optimization method of XML embedded system based on J2ME platform is proposed (5). According to the characteristics of J2ME, such as free structure, modularity and scalability, through building cross compiler environment and relevant basic configuration, operation platform of J2ME is translated and established in XML embedded system, so as to reduce the workload of system itself and improve the stability of the system. Simulation results show that, the optimization method of XML embedded system based on J2ME platform have strong stability, and high work efficiency (6).

\section{The principle of XML embedded system based on J2ME platform}

J2ME is of a part of Java developed by Sun Company, is Java operation environment of high precision, mainly for embedded consumer electronics devices such as XML. Among XML embedded system based on J2ME platform, J2ME adopts 3 layer structure design: the Java virtual machine, the Configuration layer and Profile layer. This system of modular and hierarchical structure, makes software programmed in XML embedded system based on J2ME platform suitable for different devices. (Figure 1 flow chart of XML embedded system optimization based on J2ME platform) 


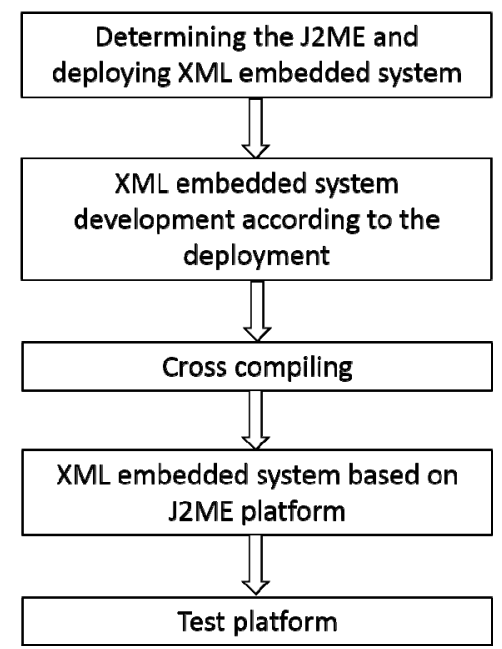

Figure 1 transplantation plan of XML embedded system based on J2ME platform

The J2ME employed in XML embedded systems have multiple performance characteristics belong to JAVA technology itself. In the XML embedded system, (product, time, place) will keep the unity through its device, and the code in XML embedded system based on the J2ME platform is random, the JAVA programming language in its own system is utilized by the XML embedded system based on the J2ME platform, network security is strong. The application programmed by the system itself is able to upgrade, and have a wide range to expand. As part of the JAVA, J2ME is similar to J2SE and J2EE, and established the complete development and deployment environment for the client and server in XML embedded system based on J2ME platform. Because only for the XML embedded system, the structure of J2ME is different from programming language and specification under the routine, which is composed of three elements, basic configuration, summary and optional package.

In the XML embedded system based on J2ME platform, J2ME technology composed of virtual machines and API, in which API provides running environment after rearranging specially in XML embedded system based on J2ME platform. J2ME mainly contains the configuration and summary. And the most prominent advantage of J2EE is to provide various services to networking (models, workstation, server), and not constrain the software of client in XML embedded system based on J2ME platform, support a relatively wide range. The detailed steps of compose is as follows:

(1) The installation of cross compilation Toolkit

Download the arm-linux-gcc-3.4.1.tar.bzc2 and extract to the directory / usr / local/arm/. Then, set the environment variable, $\sim$ edit bash_profile paths of cross compiler tool in $p A T H$ variables:

(2) Installation of J2ME

Install toolkit J2SE1.4.2, then, to set environment variables JAVA_home $=/$ usr $/$ java $/ j 2 s d k 1.4 .2 \_9$

cLASSPATH $=\$ J A V A \_H O M E /$ jre / lib/rt.jar $: \$$ java

HOME / Lib / tools.jar

Then add the path of Java tool on $p A T H$ variables:

path $=\$$ path $;$ HOME / bin;/arm 2410cl/gcc-3.4.6-glibc-2.3.6/arm -linux

arm-linux/bin\$java_home/bin

Finally, through the export to output variables at the end of the file:

exportJAVA_HOMECLASSPATH .

In general, following points can be concluded simply for in XML embedded system based on J2ME platform:

(1) In XML embedded system based on J2ME platform, the user can download the applications from Internet according to their need, without additional consumption, by the equipment manufacturer preinstalled application equipment to save the investment of users on the wireless 
device, and enable operators to strengthen and expand the service of the XML embedded system based on J2ME platform.

(2) In XML embedded system based on J2ME platform, J2ME platform provides a class library, which can make the application developers create richer and more visional graphical user interface, which also makes the service provider is capable of providing more complete diversified services in XML embedded system based on J2ME platform.

(3) In XML embedded system based on J2ME platform, J2ME platform enhance application efficiency of network bandwidth.

(4) In XML embedded system based on J2ME platform, JAVA technology provides the technical ability which can cross platform, and support for multiple devices.

(5) In XML embedded system based on J2ME platform, JAVA technology establish a potential space for the wireless device, therefore, platform across-cutting is wide. In XML embedded system based on J2ME platform, service providers can also adopt different equipment from multiple manufacturers, without supporting each device individually in XML embedded system based on J2ME platform.

\section{Optimization Analysis of XML embedded system based on J2ME platform}

In XML embedded system based on J2ME platform, there also exists some problems: slow running speed, less hardware resource of XML embedded system. So, the XML embedded system based on J2ME platform put forward the optimization method to make up for the defects mentioned above are as follows:

(1) In XML embedded system based on J2ME platform, due to the use of the self-adaptive dynamic compilation technology, the performance of the fast compiler technology is improved in XML embedded system based on J2ME platform, and its own speed is speeded up.

(2) In XML embedded system based on J2ME platform: sun realized that supporting memory and resource requirements of the full version JVM, benefit controlling the opreation of JAVA in XML embedded system, which makes developers save a lot of cost.

(3) The use of the JAVA chip XML embedded system based on the J2ME platform.

(4) Real-time JAVA technique is adopted in XML embedded system based on the J2ME platform, on the basis of reserving general reserved characteristics of JAVA, the strain and the mechanism of the latest design of XML embedded system based on the J2ME platform is increased to achieve the perfect fusion. In order to support real-time application of XML embedded system.

In summary, optimization process of XML embedded system based on J2ME platfor, reduces the workload of the system itself, and improve the stability of the system.

\section{The experiment and simulation}

In order to verify the effectiveness of optimization method for XML embedded system based on J2ME platform, there is the need for an experiment, in the process of experiment, the virtual operation environment under J2ME platform need to be built. According to the experiments results, the graph below was obtained:

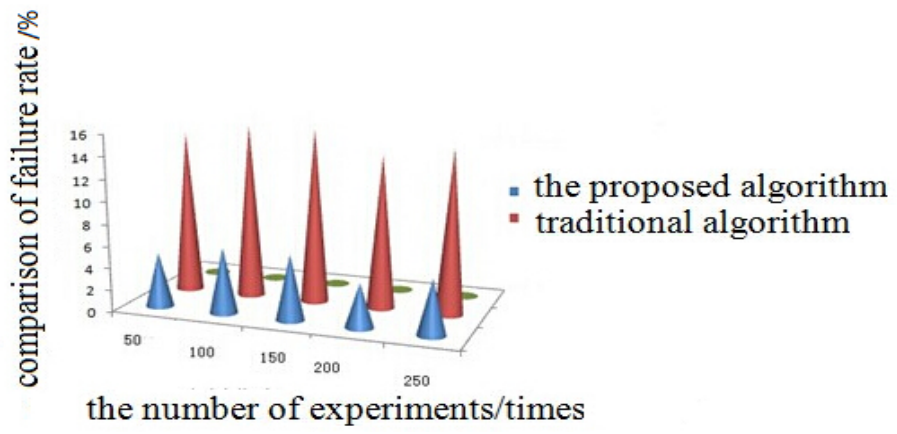

Figure 2 trend chart of fault rate comparison with different algorithms 
On the basis of the above experiments, it can be concluded that optimization method for XML embedded system based on J2ME platform has strong stability, high work efficiency.

\section{Conclusion}

In the optimization research of XML embedded system, in order to make up for the disadvantages produced by traditional method, like poor stability and time-consuming, the optimization method of XML embedded system based on J2ME platform is put forward, according to the characteristics of J2ME, such as free structure, modularity and scalability, through building cross compiler environment and relevant basic configuration, operation platform of J2ME is translated and established in XML embedded system, so as to reduce the workload of system itself and improve the stability of the system. Simulation results show that, the optimization method of XML embedded system based on J2ME platform have advantages, like high safety and good portability.

\section{Acknowledgments}

The work was supported by the first batch of education department informatization pilot units projects, with the project name Exploration of Shared Professional Teaching Resource Library construction mechanisms and application models. The other projects is Teaching reform issues of Chongqing point, Chongqing City, with the project name Research And Practice of Shared Professional Teaching Resource Library System Construction and Management.

\section{Reference}

[1] Qu Mingdi, Ma Lingzhi. The application of Java technology in embedded system [J]. Microcontroller and embedded system, 2003 (1): 14-18.

[2] Zhang Lei, Li Zhishu, Du Wei. Research and Developing Instance in J2ME [J]. Application Research of computers, 2003 (12): 158-181.

[3] Wu Yanchang, Feng Ping, Su Cong. Application Development and Research Based on J2ME Technology [J]. Microelectronics and computer, 2005, 22 (9): 71-73

[4] Liang Xu, Yu Ping. Design of Campus Information Query System Based on J2ME Platform [J]. Information Technology \& Informatization, 2011 (3): 90-92.

[5] Huang Jun, Tang Xuebo, Li Guangwei, et al. Research on J2ME mobile remote control [J]. Computer Applications and Software, 2011, 28 (7): 189-191.

[6] Deng Lianyong, Lu Yiqin. Research and implementation of Embeded GIS System Base on J2ME [J]. Control \& Automation, 2009, 25 (29): 54-56. 